\title{
Construction of the smallest common coarser of two and three set partitions
}

\author{
Radovan Potůček
}

\begin{abstract}
This paper is inspired by a text of the book [7] ("Úvod do algebry" in Czech, "Introduction to Algebra" in English) of the authors Ladislav Kosmák and Radovan Potůček. They followed the great work of Professor Otakar Borůva in the field of the partition theory, groupoids and groups and gave them in the context to contemporary modern algebra. Academian Borůvka have deduced and proved many results concerning the partition theory in his publications.

His first works [1] and [2] were published during World War II and his monographs [3] and [4] were released in the post-war years.

In this paper we deal with a construction of the smallest common coarser of two set partitions associated with equivalence relations, we give a special relation used in the construction and an illustration of blocks of this coarser.
\end{abstract}

Remark 1. Algebra of binary relations was worked up by Jacques Riguet in 1948 (see [8], the translation into Russian has its origin from 1963). Professor Borůva was rightfully proud himself on his theory of partitions and an appliance of the algebra of relations has never used. From twenty-seven chapters of the book [4] is six devoted to the general theory of partitions and in the chapter 14 and 21-24 are paragraphs dealing with partitions in the theory of grupoids and groups.

Key Words: Partition of a set, equivalence relation, smallest common coarser.

2010 Mathematics Subject Classification: 03E02; 05A18

Received: May 2013

Revised: August 2013

Accepted: October 2013 
In recent decades there has been a rapid development of the theory and applications of algebraic structures but also the algebraic hyperstructures. In this context, we may refer to interesting articles [5] of Cristine Flaut and [6] of Jan Chvalina, Šárka Hošková-Mayerová, and Dehghan Nezhad.

First, let us briefly recall some basic and well-known terms, notions and facts, without proofs, concerning partitions and equivalence relations.

Definition 1. A partition of a set $M$ is a collection $\mathcal{S}$ of nonempty subsets of $M$, called blocks, parts or cells of the partition, such that:

1. All sets in $\mathcal{S}$ are pairwise disjoint, i.e. $\forall S_{1}, S_{2} \in \mathcal{S}: S_{1} \cap S_{2}=\emptyset$ when $S_{1} \neq S_{2}$.

2. The union of all the sets forms the whole set $M$, i.e. $\bigcup_{S_{i} \in \mathcal{S}} S_{i}=M$.

Definition 2. Let $\mathcal{R}, \mathcal{S}$ be partitions of a set $M$ and let for every $X \in \mathcal{R}$ there exists such $Y \in \mathcal{S}$ that $X \subset Y$. Then the partition $\mathcal{S}$ is said to be a coarser partition than $\mathcal{R}$, the partition $\mathcal{R}$ is said to be a finer partition than $\mathcal{S}$, and we write $\mathcal{R} \sqsubset \mathcal{S}$.

If it holds $\mathcal{R} \sqsubset \mathcal{S}$, we also say that the partition $\mathcal{R}$ is a refinement of the partition $\mathcal{S}$.

Definition 3. A meet of two partitions $\mathcal{R}, \mathcal{S}$ of a set $M$, denoted by $\mathcal{R} \sqcap \mathcal{S}$, is a set of all intersections $X \cap Y$ where $X \in \mathcal{R}, Y \in \mathcal{S}$.

Definition 4. A power set of a set $M$, denoted $\mathcal{P}(M)$, is the set of all subsets of $M$, i.e. for any sets $M$ and $X$ it holds: $X \in \mathcal{P}(M)$ if and only if $X \subseteq M$.

Theorem 1. For any parts $\mathcal{A}, \mathcal{B}, \mathcal{C}$ of a set $\mathcal{P}(M)$ it holds:

1. $\mathcal{A} \sqcap \mathcal{B} \sqsubset \mathcal{A}, \mathcal{A} \sqcap \mathcal{B} \sqsubset \mathcal{B}$.

2. If $\mathcal{C} \sqsubset \mathcal{A}$ and $\mathcal{C} \sqsubset \mathcal{B}$, then $\mathcal{C} \sqsubset \mathcal{A} \sqcap \mathcal{B}$.

Theorem 2. If $\mathcal{R}, \mathcal{S}$ are partitions of a set $M$ such that $\mathcal{R} \sqsubset \mathcal{S}$ and $\mathcal{S} \sqsubset \mathcal{R}$, then $\mathcal{R}=\mathcal{S}$.

Definition 5. An equivalence relation on a set $M$ is a relation $R$ on $M$ such that:

1. $(x, x) \in R$ for all $x \in M$, i.e the relation $R$ is reflexive.

2. If $(x, y) \in R$, then $(y, x) \in R$, i.e. the relation $R$ is symmetric.

3. If $(x, y) \in R$ and $(y, z) \in R$, then $(x, z) \in R$, i.e. the relation $R$ is transitive. An equivalence class of an element $x \in M$ is defined as the set $\{y \in M ;(x, y) \in$ $R\}$. 
Remark 2. Denote an identity relation $\{(x, y) \in M \times M ; x=y\}$ by $E$, an inverse relation $\{(y, x) \in M \times M ;(x, y) \in R\}$ by $R^{-}$, and a composition of relations $R \circ R=\{(x, z) \in M \times M ; \exists y \in M:(x, y) \in R \wedge(y, z) \in S\}$ by $R^{2}$, we can write reflexivity in the form $E \subset R$, symmetry by the equality $R=R^{-}$, and transitivity as $R^{2} \subset R$.

Theorem 3. Each partition $\mathcal{R}$ of a set $M$ induces an associated equivalence relation $R$ on $M$, and conversely each equivalence relation $R$ on $M$ induces an associated partition $\mathcal{R}$ of $M$, denoted by $M / R$, into equivalences classes. Thus there is a bijection from the set of all possible equivalence relations on $M$ to the set of all partitions of $M$.

Now, let us come to our main topic:

Theorem 4. For equivalence relations $R, S$ on a set $M$ it holds $R \subset S$ if and only if $M / R \sqsubset M / S$.

Proof. Let $R \subset S$ and let $a \in M$. Then for each $x \in M$, such that $x R a$, we have $x S a$, thus $R_{a} \subset S_{a}$, i.e. $M / R \sqsubset M / S$.

When contrariwise $M / R \sqsubset M / S$, we get $R_{a} \subset S_{a}$ for each $a \in M$, and thus from $x R a$ it follows $x S a$.

Theorem 5. Let $\mathcal{R}_{1}, \mathcal{R}_{2}$ be two partitions of a set $M$ and let

$$
\mathcal{R}=\left(\mathcal{R}_{1} \sqcap \mathcal{R}_{2}\right) \backslash\{\emptyset\} .
$$

Then $\mathcal{R}$ is a partition of $M$ and it holds:

1. $\mathcal{R} \sqsubset \mathcal{R}_{1}, \mathcal{R} \sqsubset \mathcal{R}_{2}$.

2. If $\mathcal{S}$ is a partition of $M$ such that $\mathcal{S} \sqsubset \mathcal{R}_{1}$ and $\mathcal{S} \sqsubset \mathcal{R}_{2}$, then $\mathcal{S} \sqsubset \mathcal{R}$.

Proof. If $A, B \in \mathcal{R}$, then there exist sets $A_{1}, B_{1} \in \mathcal{R}_{1}, A_{2}, B_{2} \in \mathcal{R}_{2}$, such that $A=A_{1} \cap A_{2}, B=B_{1} \cap B_{2}$. If $A \neq B$, then there exists $k \in\{1,2\}$, such that $A_{k} \neq B_{k}$, so $A_{k} \cap B_{k}=\emptyset$, and thus $A \cap B=\emptyset$. By Definition 1, for any $x \in M$ there exists a block $X_{1}$ of the partition $\mathcal{R}_{1}$ and a block $X_{2}$ of the partition $\mathcal{R}_{2}$, both the blocks containing the element $x$, so $x \in X_{1} \cap X_{2} \in \mathcal{R}$. The statements 1 . and 2 . follow from Theorem 1 .

Definition 6. The partition $\mathcal{R}$ from Theorem 5 is called the greatest common refinement of the partitions $\mathcal{R}_{1}, \mathcal{R}_{2}$ and is denoted by $\mathcal{R}_{1} \wedge \mathcal{R}_{2}$.

Theorem 6. Let $\mathcal{R}_{1}, \mathcal{R}_{2}$ be two partitions of a set $M$ and let $\mathcal{S}_{0}$ is a meet of a system $\Upsilon$ of all partitions of $M$, that all are coarser than partitions $\mathcal{R}_{1}$ and $\mathcal{R}_{2}$. Then

$$
\mathcal{S}=\mathcal{S}_{0} \backslash\{\emptyset\}
$$

is a partition of $M$ and it holds: 
1. $\mathcal{R}_{1} \sqsubset \mathcal{S}, \mathcal{R}_{2} \sqsubset \mathcal{S}$.

2. If $\mathcal{T}$ is any partition of $M$ that is coarser than both the partitions $\mathcal{R}_{1}, \mathcal{R}_{2}$, then $\mathcal{S} \sqsubset \mathcal{T}$.

Proof. As $\mathcal{R}_{1} \sqsubset \mathcal{R}, \mathcal{R}_{2} \sqsubset \mathcal{R}$ for each $\mathcal{R} \in \Upsilon$, it holds, by generalized Theorem $5, \mathcal{R}_{1} \sqsubset \mathcal{S}, \mathcal{R}_{2} \sqsubset \mathcal{S}$.

If $\mathcal{T} \in \Upsilon$, then $\mathcal{T}$ is coarser then the meet $\mathcal{S}_{0}$ by its definition, so we get $\mathcal{S} \sqsubset \mathcal{T}$.

Remark 3. The partition $\mathcal{S}$ from Theorem 6 is thus the smallest common coarser of the partitions $\mathcal{R}_{1}, \mathcal{R}_{2}$. A construction of an equivalence relation, which determine this partition, is much more difficult than in a case of the greatest common refinement. We give the construction of the smallest common coarser in the following theorems.

Theorem 7. Let $R, S$ be equivalence relations on a set $M$ and let a relation $T_{R}$ on $M$ is defined this way: $(x, y) \in T_{R}$ if and only if there exists such a natural number $k$ that

$$
(x, y) \in(R S R)^{k} .
$$

Then $T_{R}$ is the equivalence relation.

Proof. Since the relation $R, S$ are reflexive, it holds

$$
E \subset(R S R)^{n}
$$

for all natural number $n$, thus $E \subset T_{R}$.

As the relation $R, S$ are symmetric, we have

$$
(R S R)^{-}=R^{-} S^{-} R^{-}=R S R,
$$

and hence $\left[(R S R)^{n}\right]^{-}=(R S R)^{n}$ for all natural numbers $n$, so $T_{R}^{-}=T_{R}$.

A transitivity of the relations $R, S$ implies that for any natural numbers $k, l$ it holds

$$
(R S R)^{k}(R S R)^{l}=(R S R)^{k+l},
$$

so the relation $T_{R}$ is transitive.

Remark 4. From a definition of the relation $T_{R}$ it follows that

$$
T_{R}=\bigcup_{k=0}^{\infty}(R S R)^{k}, \quad \text { where } \quad(R S R)^{0}=E .
$$


Theorem 8. The partition $\mathcal{T}$ determined by the equivalence relation $T_{R}$ from Theorem 7 is a common coarser of the partitions $\mathcal{R}, \mathcal{S}$ associated with the equivalence relations $R, S$.

Proof. The reflexivity of the relations $R, S$ implies

$$
\begin{gathered}
R=R E \subset R S=R S E \subset R S R \subset(R S R)^{n} \subset T_{R}, \\
S=E S E \subset R S R \subset(R S R)^{n} \subset T_{R}
\end{gathered}
$$

for all natural numbers $n$. Hence $R \subset T_{R}$ a $S \subset T_{R}$, and by Theorem 2 we have

$$
\mathcal{R} \sqsubset \mathcal{T}, \quad \mathcal{S} \sqsubset \mathcal{T} .
$$

Theorem 9. At a notation used in Theorem 8, let $\mathcal{V}$ is an arbitrary common coarser of the partitions $\mathcal{R}, \mathcal{S}$. Then

$$
\mathcal{T} \sqsubset \mathcal{V},
$$

hence $\mathcal{T}$ is the smallest common coarser of the partitions $\mathcal{R}, \mathcal{S}$.

Remark 5. For the partition $\mathcal{T}$ from Theorem 9 we shall use a notation $\mathcal{R} \vee \mathcal{S}$.

Proof. If $V$ is an equivalence relation associated with the partition $\mathcal{V}$, then it holds $R \subset V, S \subset V$, thus for all natural numbers $k$ we have

$$
(R S R)^{k} \subset V^{3 k} \text {. }
$$

A transitivity of the relation relace $V$ implies that

$$
V \supset V^{2} \supset V^{3} \supset \cdots,
$$

so

$$
T_{R}=\bigcup_{k=0}^{\infty}(R S R)^{k} \subset \bigcup_{k=0}^{\infty} V^{k}=V .
$$

Hence $T_{R} \subset V$, and by Theorem 2 we get $\mathcal{T} \sqsubset \mathcal{V}$. This proves that

$$
\mathcal{T}=\mathcal{R} \vee \mathcal{S} .
$$

Theorem 10. Let $R, S$ be equivalence relations on a set $M$ and let a relation $T_{S}$ on $M$ is defined this way: $(x, y) \in T_{S}$ if and only if there exists such a natural number $k$ that

$$
(x, y) \in(S R S)^{k} .
$$

Then $T_{S}=T_{R}$. 
Proof. The inclusions

$$
\begin{aligned}
& R S R \subset S(R S R) S=(S R S)(S R S) \subset S R S, \\
& S R S \subset R(S R S) R=(R S R)(R S R) \subset R S R
\end{aligned}
$$

imply the equality

$$
R S R=S R S,
$$

and hence

$$
T_{S}=\bigcup_{k=0}^{\infty}(S R S)^{k}=\bigcup_{k=0}^{\infty}(R S R)^{k}=T_{R}
$$

Corollary 1. Both equivalence relations $T_{R}, T_{S}$ determine the same partition $\mathcal{R} \vee \mathcal{S}$.

Remark 6. Now, we show a representation of the relation $R S R$, where $A_{1}, A_{2}, A_{3}$ are blocks of the partition $\mathcal{R}$ and $B_{1}, B_{2}$ are blocks of the partition $\mathcal{S}$, and a construction of the smallest common coarser of two partitions. In the picture 1.1 below is schematically presented a relation

$$
a_{1} R c_{1} S c_{2} R a_{2} R c_{3} S c_{4} R a_{3}, \quad \text { i.e. } \quad a_{1} R S R a_{2} R S R a_{3}, \quad \text { i.e. } \quad a_{1}(R S R)^{2} a_{3} .
$$

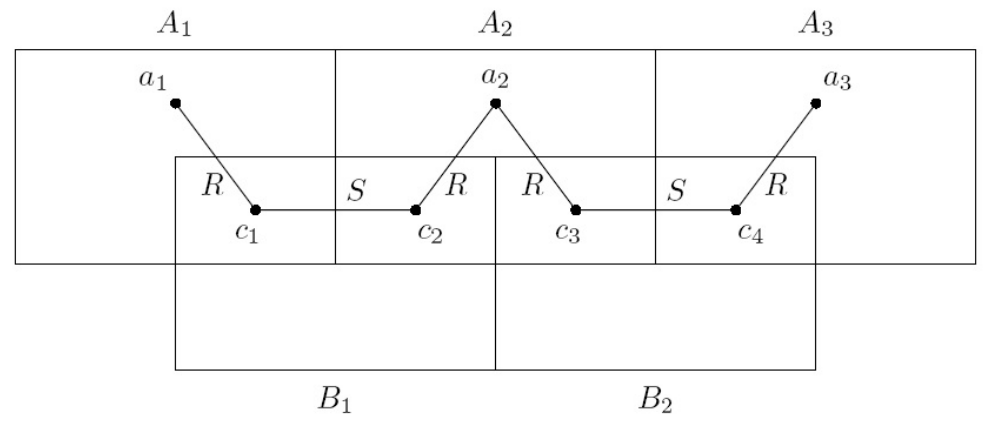

Pic. 1.1

In the picture 1.2 a set $M$ is the rectangle $P_{1} P_{2} P_{3} P_{4}, A_{1}, \ldots, A_{4}$ are blocks of the partition $\mathcal{R}, B_{1}, \ldots, B_{6}$ are blocks of the partition $\mathcal{S}$, and $A_{1} \cup A_{2}, A_{3} \cup A_{4}$ are blocks of the smallest common coarser $\mathcal{R} \vee \mathcal{S}$. Two blocks of the smallest common coarser are also presented: 


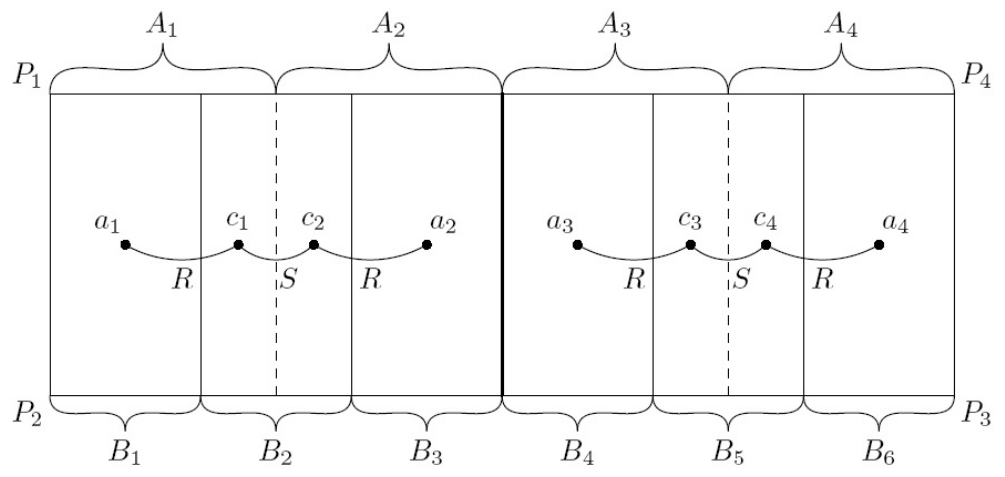

Pic. 1.2

Theorem 11. Let $R, S, T$ be equivalence relations on a set $M$ and let a relation $V_{1}$ on $M$ is defined this way: $(x, y) \in V_{1}$ if and only if there exists such a natural number $k$ that

$$
(x, y) \in(R S T S R)^{k} .
$$

Then $V_{1}$ is the equivalence relation.

Proof. Since the relations $R, S, T$ are reflexive, it holds

$$
E \subset(R S T S R)^{n}
$$

for all natural number $n$, thus $E \subset V_{1}$.

As the relations $R, S, T$ are symmetric, we have

$$
(R S T S R)^{-}=R^{-} S^{-} T^{-} S^{-} R^{-}=R S T S R,
$$

and hence

$$
\left[(R S T S R)^{n}\right]^{-}=(R S T S R)^{n} .
$$

If there exist such natural numbers $k, l$, that

$$
\begin{aligned}
& (x, y) \in(R S T S R)^{k}, \\
& (y, z) \in(R S T S R)^{l},
\end{aligned}
$$

then

$$
(x, y) \in(R S T S R)^{k}(R S T S R)^{l}=(R S T S R)^{k+l},
$$

so the relation $V_{1}$ is transitive. 
Theorem 12. Let $R, S, T$ be equivalence relations on a set $M$ and let a relation $V_{2}$ on $M$ is defined this way: $(x, y) \in V_{2}$ if and only if there exists such a natural number $k$ that

$$
(x, y) \in(T S R S T)^{k} .
$$

Then $V_{2}$ is the equivalence relation.

Proof. Is similar to the proof of Theorem 11, where the relations $R$ and $T$ are mutually replaced.

Theorem 13. At a notation used in Theorems 11 and 12 it holds

$$
V_{1}=V_{2}
$$

Proof. The inclusions

$$
\begin{aligned}
& T S R S T \subset R S(T S R S T) S R \subset(R S T S R)(R S T S R) \subset R S T S R \\
& R S T S R \subset T S(R S T S R) S T \subset(T S R S T)(T S R S T) \subset T S R S T
\end{aligned}
$$

imply the equality

$$
R S T S R=T S R S T
$$

and hence

$$
V_{1}=\bigcup_{k=0}^{\infty}(R S T S R)^{k}=\bigcup_{k=0}^{\infty}(T S R S T)^{k}=V_{2} .
$$

Theorem 14. The partition $\mathcal{V}$ determined by the equivalence relations $V_{1}, V_{2}$ is the smallest common coarser of the partitions $\mathcal{R}, \mathcal{S}, \mathcal{T}$ associated with the equivalence relations $R, S, T$.

Proof. We have

$$
R \subset R S \subset R S T \subset R S T S \subset R S T S R \subset V_{1}
$$

and similarly $S \subset V_{1}, T \subset V_{1}$, so the partition $\mathcal{V}$ is the common coarser of the partitions $\mathcal{R}, \mathcal{S}, \mathcal{T}$.

If $\mathcal{W}$ is an arbitrary common coarser of the partitions $\mathcal{R}, \mathcal{S}, \mathcal{T}$ and if $W$ is an equivalence relation which determines this partition, then they hold the inclusions

$$
R \subset W, \quad S \subset W, \quad T \subset W,
$$

and hence for every natural number $n$ we get

$$
(\operatorname{RSTSR})^{n} \subset W^{5 n} .
$$


Since the relation $W$ is transitive, we have

$$
W \supset W^{2} \supset W^{3} \supset \cdots,
$$

and so

$$
V=\bigcup_{k=1}^{\infty}(R S T S R)^{k} \subset W \cup W^{2} \cup W^{3} \cup \cdots=W .
$$

By Theorem 4, we have proved that $\mathcal{V}$ is the smallest common coarser of the partitions $\mathcal{R}, \mathcal{S}, \mathcal{T}$.

Remark 7. Denote by $\mathcal{R} \vee \mathcal{S}$ the smallest common coarser of the partitions $\mathcal{R}, \mathcal{S}$, we obtain the formulas

1. $\mathcal{R} \vee \mathcal{S}=\mathcal{S} \vee \mathcal{R}$,

2. $\mathcal{R} \vee(\mathcal{S} \vee \mathcal{T})=(\mathcal{R} \vee \mathcal{S}) \vee \mathcal{T}$.

as the results of Theorems 10 and 13 .

At the proof of the proposition 2., it was useful to apply the equality

$$
(\mathcal{R} \vee \mathcal{S}) \vee \mathcal{T}=\mathcal{T} \vee(\mathcal{R} \vee \mathcal{S})
$$

Theorem 15. For arbitrary partitions $\mathcal{R}, \mathcal{S}$ of a set $M$ they hold the equalities

$$
\begin{aligned}
& \mathcal{R} \vee(\mathcal{R} \wedge \mathcal{S})=\mathcal{R}, \\
& \mathcal{R} \wedge(\mathcal{R} \vee \mathcal{S})=\mathcal{R} .
\end{aligned}
$$

Proof. Both propositions of the theorem follow from the relations

$$
\begin{aligned}
\mathcal{R} \wedge \mathcal{S} & \sqsubset \mathcal{R}, \\
\mathcal{R} & \sqsubset \mathcal{R} \vee \mathcal{S} .
\end{aligned}
$$

Acknowledgements: The publication of this article was partially supported by the grant PN-II-ID-WE-2012-4-169 of the Workshop "A new approach in theoretical and applied methods in algebra and analysis".

\section{References}

[1] Borůvka, O., Theorie grupoidů, část první. Spisy vyd. Př́rodovědeckou fakultou Masarykovy University, č. 275, Brno 1939.

[2] Borůvka, O., Úvod do theorie grup. 1. vydání, Praha 1944.

[3] Borůvka, O., Úvod do theorie grup. 2. vydání, Přírodovědecké vydavatelství, Praha 1952. 
[4] Borůvka, O., Základy teorie grupoidi̊ a grup. Nakladatelství ČSAV, Praha 1962.

[5] Flaut, C., Division algebras with dimension $2^{\wedge} t$, where $t$ belongs to $N$. Analele Stiintifice ale Universitatii Ovidius Constanta, Seria Matematica, 13(2)(2005), 31-38.

[6] Chvalina, J., Hošková-Mayerová, Š., Nezhad, A., D., General actions of hyperstructures and some applications. Analele Stiintifice ale Universitatii Ovidius Constanta, Seria Matematica, 21(1)(2013), 59-82.

[7] Kosmák, L., Potůček, R., Úvod do algebry. KEY Publishing, Ostrava 2012. ISBN 978-80-7418-162-7.

[8] Riguet, J., Relations binaires, fermetures, correspondances de Galois. Bull. Soc. Math. France 76 (1948), 1-4, 114-155 (Russian translation: Binarnyje otnošenija, zamykanija, sootvetstvija Galua, Kibernetičeskij sbornik 7 (1963), 129-185).

Radovan POTU゚ČEK,

Faculty of Military Technology,

University of Defence,

Kounicova 65, 66210 Brno, Czech Republic

E-mail: Radovan.Potucek@unob.cz 